\title{
Reaktionskraftuntersuchungen bei Vorschub und Rückzug von PTCA-Kathetern in modellierten Koronargefäßen
}

\author{
Kraft M., Rutsch W.*, Boenick U. \\ Institut für Mikrotechnik und Medizintechnik, Technische Universität Berlin \\ * Zentrum für Innere Medizin, Medizinische Klinik und Poliklinik I, Charité, Humboldt Universität Berlin
}

\section{Einleitung}

PTCA-Ballonkatheter werden zur Aufweitung von Verengungen der Herzkranzgefäße genutzt. Sic finden in verschiedenen Bauarten Anwendung. Zwischen diesen Kathetertypen und den Kathetern einer Bauart aber verschiedener Hersteller bestehen deutliche Differenzen in der Ausprägung anwendungsrelevanter Kathetermerkmalc.

In einem ncuen Prüfstand für PTCA-Katheter [1,2] werden der anatomische Aufbau (Lage, Durchmesser, Verzweigungen der Gefäße) und die Reibverhältnisse den physiologischen Verhältnissen nachgebildet. Möglich sind Messungen von Aktionskräften am Antrieb kombiniert mit Reaktionskraftmessungen in Modellgefäßen [1]. Innerhalb dicses Beitrages werden Untersuchungen vorgestellt, die neben Abstützkräften des Kathetcrs im Führungskatheter auch auf die Gefäßwand übertragene Kräfte bei Passage cines gekrümmten Gefäßabschnittes erfassen.

\section{MATERIAL UND METHODE}

Die Kraftmessungen wurden an sterilen, für den klinischen Gebrauch bestimmten PTCA-Kathetern der Typen SCIMED ${ }^{3}$ Viva $^{\text {TM }}$ und Long Viva ${ }^{\mathrm{TM}}$ (Fa. Boston Scientific Corp.), Bonnie ${ }^{\text {TM }}$ (Fa. Schneider AG) und ACS $\mathrm{RX}^{\mathbb{E}}$ Rocket $^{\mathrm{TM}}$ (Fa. Guidant), alle mit Ballonabmessungen $3,0 \mathrm{~mm} / 20 \mathrm{~mm}$ bis auf Long Viva ${ }^{\mathrm{TM}}$ mit $3,0 / 30 \mathrm{~mm}$, durchgeführt. Vor Einbringung in das Modellgefäß sind alle Katheter für $15 \mathrm{~s}$ aspiriert worden.

Das verwendete PE-Modellgefäß simuliert einen rechten Gefäßbaum mit der anatomischen Besondcrheit des Vorhandenseins einer proximalen Krümmung. Aus Abb. 1 ist die Lage der Krümmungen im Modellgefäß crsichtlich. Die Ergebnisdiagramme (Abb. 2-7) enthalten ebenfalls entsprechende Bczeichnungen. Die Katheter wurden $90 \mathrm{~mm}$ tief in das Modell eingebracht. Als Meßstrecke diente der Abschnitt zwischen $20 \mathrm{~mm}$ und $90 \mathrm{~mm}$. Verwendete Kraftaufnehmer befinden sich am Antrieb [2], am distalen Ende des Führungskatheters und in der 3. Krümmung des Gefäßsystems $(65 \mathrm{~mm}$ nach Beginn der Mcßstrecke). Alle Kraftaufnehmer sind als AluminiumBiegebalken (Wirklänge $28 \mathrm{~mm}$ ) mit DoppelgitterDehnungsmeßstreifen (Vollbrücke) bestückt.

Verwendete Zubehörkomponenten waren ein Y-Konnektor mit hämostatischem Adapter, ein $7 F$ JR 4,0 SH Führungskatheter und ein 0,014 inch $\times 175 \mathrm{~cm}$ SoftFührungsdraht (beide Fa. Asahi Intecc Co., Japan). Die Vorschub- und Rückzuggeschwindigkeit betrug $4 \mathrm{~mm} / \mathrm{s}$. Das Gefäßmodell ist vor jeder Messung über eine Spülung mit destilliertem Wasser auf $37 \pm 1^{\circ} \mathrm{C}$ tempericrt worden. Mit jedem Katheter (je 2 Stück der 0.g. Hersteller) wurden 3 Messungen der Aktions- und Reaktionskräfte vorgenommen. Die verwendete Abtastfrequenz lag über $80 \mathrm{~Hz}$. Eine erste Datenaufbereitung beinhaltcte eine
Reduktion der Stützstellen durch Mittelwertbildung auf 2 Wertc pro Millimeter Weglängc. Zur Veranschaulichung der Ergebnisse crfolgte eine weitere Zusammenfassung durch Mittelwertbildung aus den Messungen eines Kathetcrtyps (6 pro Typ).

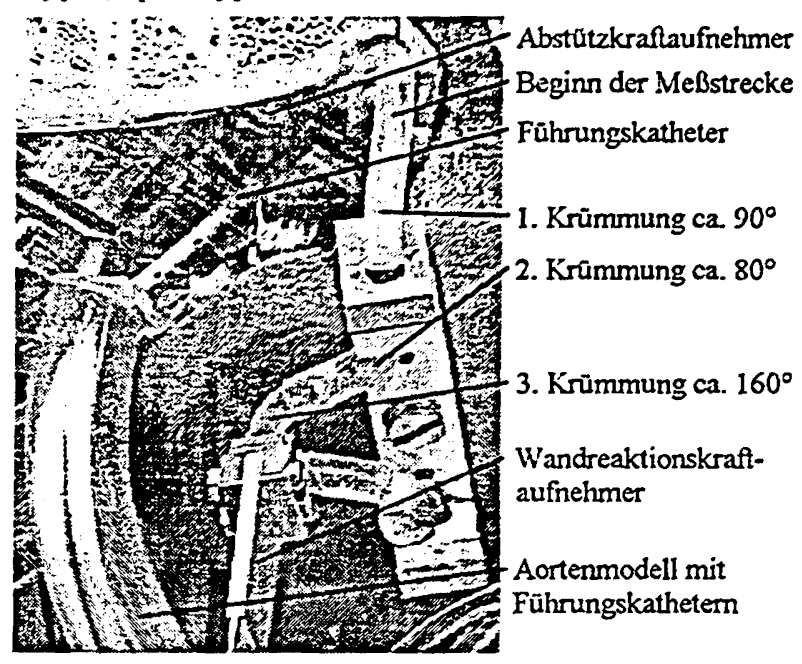

Abb. 1: Lage der Krümmungen im Gefäßmodell (RCA)

\section{ERGEBNISSE}

Die nachfolgenden Abbildungen 2-7 enthalten die Resultate der durchgeführten Messungen. Die Reaktionskräfte wurden zur Verdeutlichung um den Faktor 10 vergrößert dargestellt (Meßbereich bis 0,04N). Vorschubkräfte, Abstützkräfte in Richtung Ostium (Zug am Führungskatheter) und Wandreaktionskräfte in Richtung Mittelpunkt des Kreissegmentes wurden positiv, entgegengesetzt wirkende Kräfte negativ aufgetragen.

In allen Messungen ist ein deutlicher Zusammenhang, fast eine Proportionalität, zwischen gemessener Vorschubbzw. Rückzugkraft und der Abstützkraft im Führungskatheter erkennbar. Diese Abhängigkeit besteht sehr ausgeprägt bei den hier untersuchten, noch industriell gefalteten.Ballons. In weiteren, in diesem Beitrag nicht im Detail vorgestellten Messungen am bereits aufgeweiteten Ballon existierte sie nur im proximalen Gefäßabschnitt. Weiter distal steigt in diesem Fall die Abstützkraft weniger stark an, da der Katheter vermutlich durch crhöhte Reibung im Gefäß eines geringeren Backups im Führungskatheter bedarf. Er stützt sich wahrscheinlich schon im Ballonbereich an der Gefäßwand ab. Mit der Erfassung von Abstützkräften im Führungskatheter wird ein wichtiges Kathetermerkmal deutlich, da unter ungünstigen Verhältnissen letztlich eine Deplazierung des Führungskatheters aus dem Ostium verursacht werden kann.

Bei der Analyse der Wandreaktionskräfte fällt auf, daß bereits lange vor Eintritt des PTCA-Katheters in die Gefäßkrümmung geringe Kräfte in Richtung Innenseite 


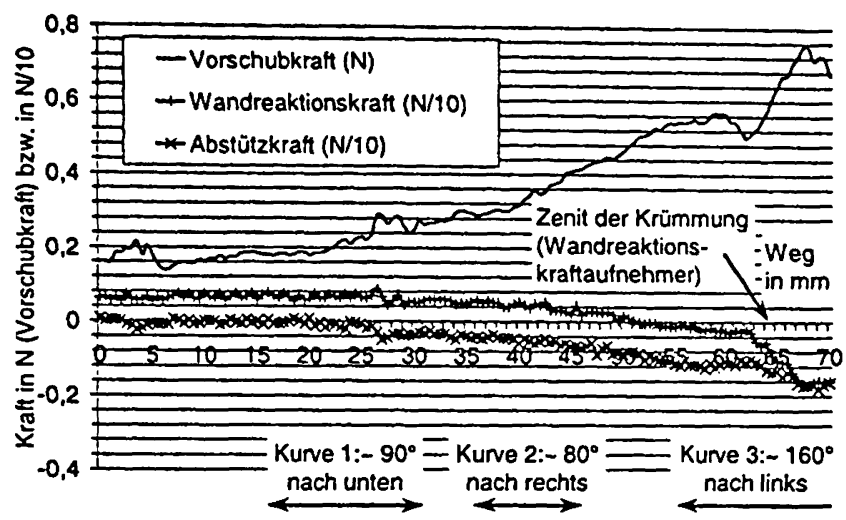

Abb. 2: Schneider Bonnie Vorschub

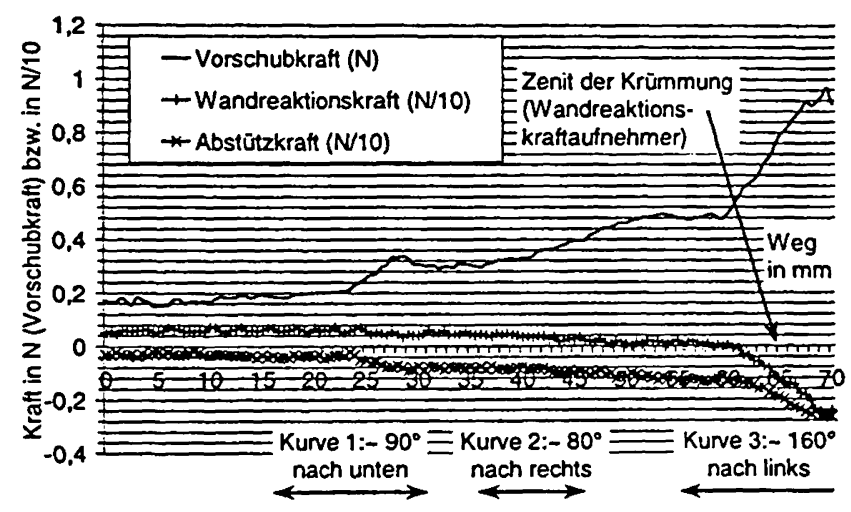

\section{Abb.3: Scimed Viva Vorschub}

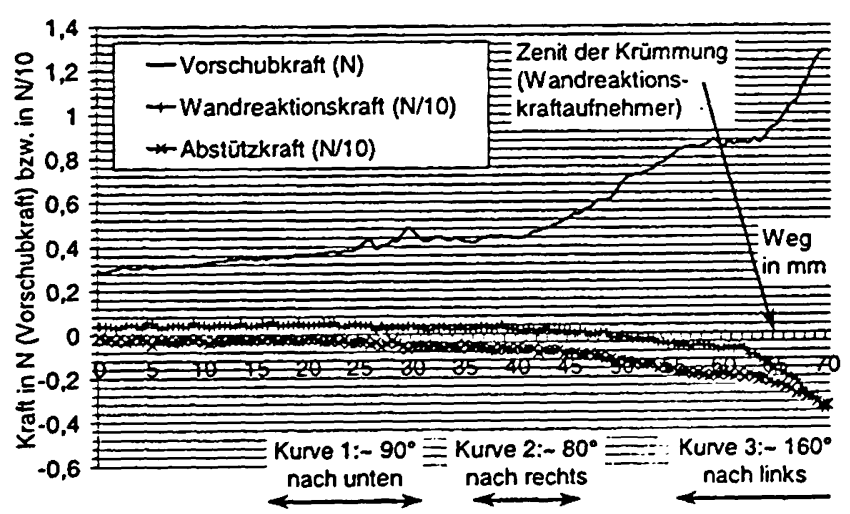

\section{Abb. 4: ACS Rocket Vorschub}

auftreten $(\approx 0,01 \mathrm{~N})$. Sie werden sehr wahrscheinlich durch den Führungsdraht verursacht. Aufgrund seiner geringen, aber vorhandenen Steifigkeit liegt er vor Ankunft des PTCA-Katheter an der Innenseite der Gefäßwand an und drückt sie in Richtung Krümmungsmittelpunkt. So ist weiterhin die gleich große Wandreaktionskraft nach Rückzug des Katheter aus der Krümmung erklärbar.

Ein Maximum der Wandreaktionskraft bei Vorschub des PTCA-Katheters tritt auf, nachdem die Katheterspitze bereits rund 4mm über die Position des Aufnehmers (siehe Abb. 1) hinaus geschoben wurde. Ursache ist vermutlich die erst hier eintretende Biegung des Katheters, nachdem er zuvor von der Innen- zur Außenseite des Modellgefäßes (Durchmesser 3,5mm) wechselte. Das Maximum der Vorschubkraft tritt bei der gleichen Katheterposition auf.

Das späte Maximum der gemessenen Rückzugkraft erklärt sich auch aus dem Seitenwechsel des Katheters (Gefäß-

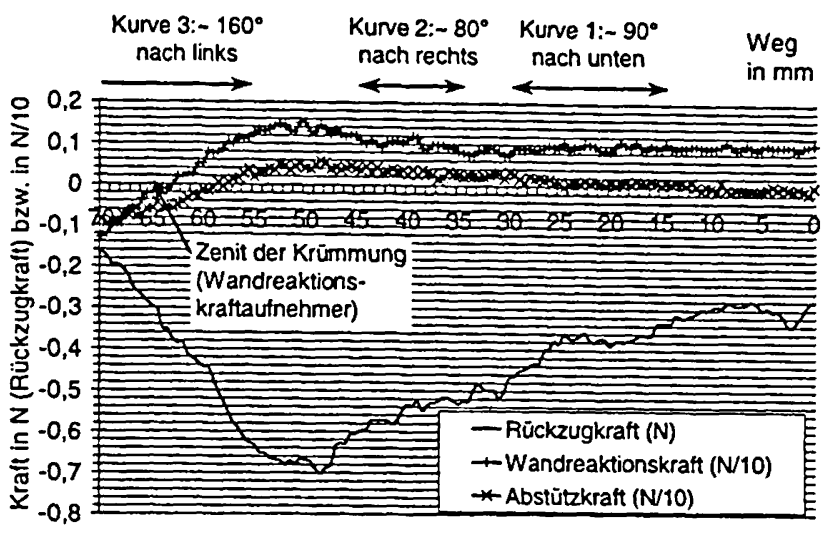

Abb. 5: Schneider Bonnie Rückzug

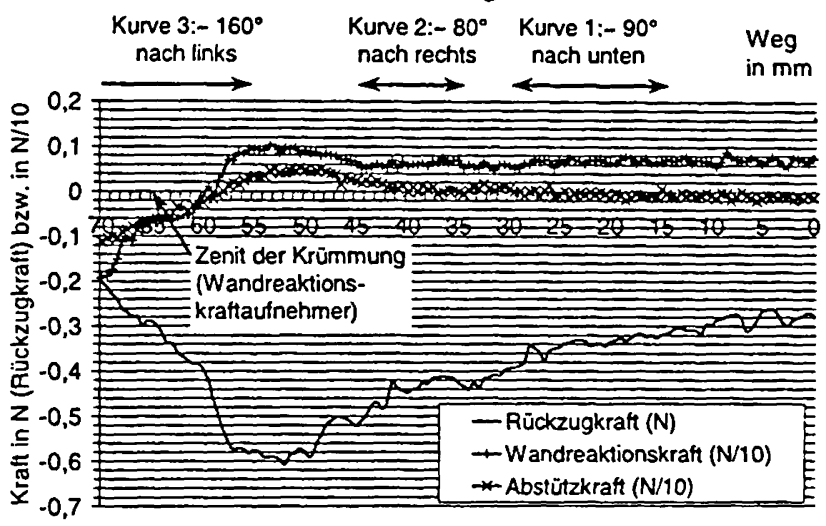

Abb. 6: Scimed Viva Rückzug

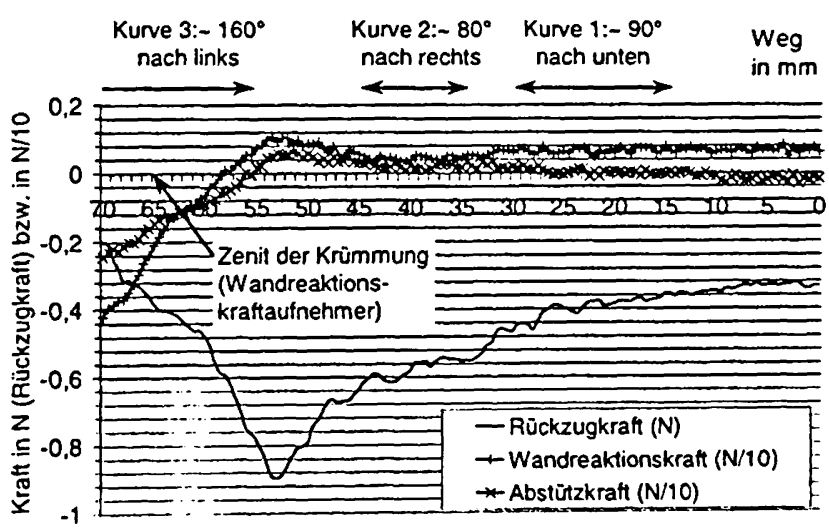

Abb. 7: ACS Rocket Rückzug

außenwände in Krümmungen bei Vorschub, Gefäßinnenwände bei Rückzug). Über die ersten $15 \mathrm{~mm}$ bis $20 \mathrm{~mm}$ erfolgt also im wesentlichen eine Straffung, ohne effektiven Rückzug.

An anderer Stelle werden weitere bereits durchgeführte Reaktionskraftmessungen der Kraftübertragung auf den Führungsdraht und zum Recrossingverhalten vorgestellt.

\section{LITERATUR}

[1] Kraft, M.; Rutsch, W.; Boenick, U.: Ein multifunktionales Herzkranzgefäßmodell: Nutzungsvarianten für vergleichende Untersuchungen an PTCA-Kathetern, Zcitschrift für Biomed. Technik, Band 43 (Erg.band). 1998

[2] Kraft, M.; Wendt, O.; Rutsch, W.; Bocnick, U.: Realisierung eincs Katheterantriebes und der Mießwerterfassung in einem Herzkranzgefaßmodell für PTCAKatheter, Zeitschrift für Biomed. Technik, Band 42 (Erg.band), 1997, S. 484-485 